cond-mat/9305007, submitted to Phys. Rev. B

\title{
Electronic Structure and Bonding in Epitaxially Stabilized Cubic Iron Silicides
}

\author{
Kurt A. Mäder甘 and Hans von Känel \\ Laboratorium für Festkörperphysik, Eidgenössische Technische Hochschule Hönggerberg, CH-8093 \\ Zürich, Switzerland \\ and \\ Alfonso Baldereschi过 \\ Institut Romand de Recherche Numérique en Physique des Matériaux, Ecublens, CH-1015 \\ Lausanne, \\ and Institut de Physique Appliquée, Ecole Polytechnique Fédérale Lausanne, CH-1015 Lausanne, \\ Switzerland.
}

(Received: 1 February 1993)

\begin{abstract}
We present an $a b$ initio full-potential linearized augmented plane-wave (FLAPW) study of the structural and electronic properties of the two bulk unstable compounds $\mathrm{FeSi}$ ( $\mathrm{CsCl}$ structure) and $\mathrm{FeSi}_{2}\left(\mathrm{CaF}_{2}\right.$ structure) which have recently been grown by molecular beam epitaxy on $\operatorname{Si}(111)$. We obtain equilibrium bulk lattice constants of $2.72 \AA$ and $5.32 \AA$ for $\mathrm{FeSi}$ and $\mathrm{FeSi}_{2}$, respectively. The density of states (DOS) of FeSi agrees well with experiment, and shows metallic behavior. In agreement with a previous calculation the DOS of $\mathrm{FeSi}_{2}$ shows a large density of $d$-states at the Fermi level, explaining the instability of the bulk phase. The electron charge distributions reveal a small charge transfer from Si to Fe atomic spheres in both compounds. While in $\mathrm{FeSi}$ the Fe-Si bond is indeed partially ionic, we show that in $\mathrm{FeSi}_{2}$ the electron distribution corresponds to a covalent charge accumulation in the $\mathrm{Fe}-\mathrm{Si}$ bond region. The reversed order of $d$-bands in $\mathrm{FeSi}$ with respect to $\mathrm{FeSi}_{2}$ is understood in terms of crystal field splitting and Fe-Fe nearest neighbor $d d$-interactions in the $\mathrm{CsCl}$ structure, and a strong $\mathrm{Si} p / \mathrm{Fe} d$ bonding in the fluorite structure, respectively.
\end{abstract}




\section{INTRODUCTION}

The current interest in transition-metal silicides stems from both fundamental and technological issues. For applications in microelectronics and optoelectronics the main focus is on silicides which can be grown epitaxially on $\mathrm{Si}$ such as, e.g., $\mathrm{NiSi}_{2}$ and $\mathrm{CoSi}_{2}$ whose small lattice mismatch with Si allows for the synthesis of high quality semiconductor/metal heterostructures1. These systems open the way for the study of intriguing fundamental questions, such as Schottky barrier formation, interface structure, growth kinetics, and stability of novel epitaxial phases. Whereas the $\mathrm{CaF}_{2}$ structure of both $\mathrm{CoSi}_{2}$ and $\mathrm{NiSi}_{2}$ are stable bulk phases of these compounds, $\mathrm{FeSi}_{2}$ does not crystallize in the fluorite form. At this stoichiometry and below $\sim 1000^{\circ} \mathrm{C}$ its stable bulk form is 3 3 $\beta$-FeSi $i_{2}$, which is semiconducting. This orthorhombic structure can be regarded as a distorted fluorite structure, and its greater stability with respect to the fluorite phase can be understood in terms of the electronic structure of the latter: a high density of $d$ states ( $d$-DOS) at the Fermi level $E_{F}$ leads to a Jahn-Teller-like instability of fluorite $\mathrm{FeSi}_{2}$. In $\beta$-FeSi ${ }_{2}$ the $d$ levels are crystal field split at $E_{F}$, and the opening of a gapt $\left(E_{\mathrm{g}} \approx 0.8 \mathrm{eV}\right)$ leads to semiconducting behavior. At composition 1:1 in the Fe-Si phase diagram 3 we find the compound $\epsilon$-FeSi. This structure is actually referred to as the "FeSi" structure, and its Pearson symbol is $c P 8$.

Under the growth conditions of molecular beam epitaxy (MBE) on $\mathrm{Si}(111)$ the Fe-Si phase diagram is dramatically different国: at a $\mathrm{Fe} / \mathrm{Si}$ composition ratio of $1: 2$ not only epitaxial $\beta-\mathrm{FeSi}_{2}$ is found, but also the bulk unstable fluorite phase, denoted $\gamma-\mathrm{FeSi}_{2}$ in Ref. 6. At composition $1: 1$ besides $\epsilon$-FeSi the $\mathrm{CsCl}$ structure of $\mathrm{FeSi}$ grows pseudomorphically on $\mathrm{Si}(111)$. Hence we deal with two novel materials completely absent in the bulk phase diagram. Before turning to the electronic and structural properties of these two structures, let us briefly discuss the physical mechanisms pertinent to the occurrence of novel epitaxial phases. (We shall use the term "epitaxial phase" only for novel phases which do not exist in bulk form, in order to emphasize their distinctiveness from stable bulk phases which may also be present in epitaxial phase diagrams.)

We distinguish two classes of epitaxial phases: $(i)$ epitaxially stable phases, where the strain conditions imposed by the substrate select a species with a lower epitaxial formation enthalpy than the strained bulk phase at that particular substrate lattice constant (ii) epitaxially metastable phases, where kinetic barriers inhibit the transformation to a (epitaxially) more stable phase.

For 1:1 FeSi, we argue that up to a critical film thickness the $\mathrm{CsCl}$ structure of $\mathrm{FeSi}$ belongs to class $(i)$, i.e., it is epitaxially stable on $\operatorname{Si}(111)$. Experimentally it is found that low temperature MBE growth of $\mathrm{FeSi}$ on $\mathrm{Si}(111)$ yields coherent films in the $\mathrm{CsCl}$ structure up to $\sim 100 \AA$, above which the creation of misfit dislocations leads to partial relaxation. Films thicker than $15 \AA$ transform into relaxed $\epsilon$-FeSi upon annealing at $\sim 300^{\circ} \mathrm{C}$. The experimentally determined lattice mismatches with $\mathrm{Si}(111)$ are $+2 \%$ for $\mathrm{FeSi}(\mathrm{CsCl})$ and $-6.4 \%$ for $\epsilon$ - $\mathrm{FeSi}$, respectively. We can rationalize these experimental findings as follows (see Fig. (1): Due to the large lattice mismatch, pseudomorphic $\epsilon$-FeSi will have a very high strain energy, and a small critical thickness $h_{1}$, whereas the much smaller mismatch between

$\mathrm{FeSi}(\mathrm{CsCl})$ and the $\mathrm{Si}$ substrate makes this material more stable at small thickness. Since $\epsilon$-FeSi is the stable bulk phase, however, above a critical thickness $H_{c}$ where it is completely relaxed, it is lower in energy than the $\mathrm{CsCl}$ structure at any thickness. Therefore, at some 
thickness $h_{2}$ the two energy curves cross, and the $\mathrm{CsCl}$ structure ceases to be the epitaxially stable phase. From experiment we infer $h_{2} \lesssim H_{c} \approx 15 \AA$. Due to kinetic barriers (e.g., an energetically favorable interface structure), the $\mathrm{CsCl}$ phase is observed in metastable form even above $h_{2}$, and beyond its critical thickness $h_{3} \approx 100 \AA$ up to $\sim 1000 \AA$.

At composition ratio 1:2 the situation is less clear. Many epitaxial relations for $\beta$ $\mathrm{FeSi}_{2} / \mathrm{Si}(111)$ with different lattice mismatches are reported 19 , and a disordered $\mathrm{Fe}_{1-x} \square_{x} \mathrm{Si}$ phase of $\mathrm{CsCl}$ symmetry with an iron vacancy concentration $x=0.5$ acts as a precursor to the ordered $\mathrm{CaF}_{2}$ phased. We will show, however, that the $\mathrm{CaF}_{2}$ phase is even better lattice matched to $\mathrm{Si}$ than the $\mathrm{CsCl}$ phase, and hence a similar picture as sketched in Fig. 11 may apply also in this case.

Whereas the existence of epitaxial fluorite $\mathrm{FeSi}_{2}$ is not that surprising in view of its relation to the bulk stable $\beta$-phase and the existence of $\mathrm{CoSi}_{2}$ and $\mathrm{NiSi}_{2}$ in this crystal structure, the $\mathrm{CsCl}$ structure of FeSi seems to be rather "exotic". It is interesting to note that the only transition metal silicides with composition $M \mathrm{Si}$ which occur in the $\mathrm{CsCl}$ structure are those with $113=\mathrm{Ru}$, Os, and Rh. Ruthenium and Rhodium are the transition metals situated in the Pd row of the periodic table just below Fe and Co, respectively. These silicides normally order in the "FeSi" structure type, i.e., the structure of $\epsilon$ - $\mathrm{FeSi}$, and only under certain growth conditions do they form the $\mathrm{CsCl}$ structure1 1 . For instance, in the case of $\mathrm{RuSi}$ it was observed that the $\mathrm{CsCl}$ structure formed "more easily in mixtures that had not yet reached equilibrium" 1 . We have argued above that FeSi films are epitaxially metastable above $\sim 15 \AA$, and only grow under the out-of-equilibrium conditions of low temperature MBE. These considerations suggest that although the $\mathrm{CsCl}$ structure is extremely rare for mono-silicides, it is nevertheless most likely to be formed by certain group VIII transition metals, among which we find Fe and Co. Indeed, in Villars' three-dimensional structural stability diagram 2 , both compounds are found in regions of coexistence of the $\mathrm{MnP}-\mathrm{FeSi}-$ $\mathrm{CsCl}$ structure types, but almost on the boundary with the region occupied by the $\mathrm{CsCl}$ structure. Very recently, epitaxial growth of CsCl-type CoSi on $\mathrm{Si}(111)$ has indeed been accomplished by $\mathrm{MBE}^{3}$. The existence of $\mathrm{CsCl}$-type NiSi, however, seems less probable.

The great versatility of $\mathrm{Fe}$ to form a number of epitaxial silicides on $\mathrm{Si}$-among which are found metals $\left(\mathrm{FeSi}(\mathrm{CsCl}), \gamma-\mathrm{FeSi}_{2}\right)$, semiconductors or semimetals $\left(\beta-\mathrm{FeSi}_{2}, \epsilon-\mathrm{FeSi}\right)$, and even magnetic compounds $\left(\mathrm{Fe}_{3} \mathrm{Si}\right.$, possibly $\left.\gamma-\mathrm{FeSi}_{2}\right)$ - offers a wide spectrum of potential applications. Theoretical studies of the electronic and structural properties of the Fe silicides are therefore of great interest. Semiconducting $\beta-\mathrm{FeSi}_{2}$ has been studied by the linear muffin-tin orbital (LMTO) the electronic structure of the fluorite phase $\gamma-\mathrm{FeSi}_{2}$ is also given. The magnetic compound $\mathrm{Fe}_{3} \mathrm{Si}\left(D O_{3}\right.$ structure) has been studied theoretically by Kudrnovský et al. 5 . We have recently published a brief account of the electronic band structure of $\mathrm{FeSi}$ in the $\mathrm{CsCl}$ structure, together with experimental results

In this work we concentrate on the properties of the bulk unstable "high symmetry" compounds $\mathrm{FeSi}$ ( $\mathrm{CsCl}$ structure) and $\gamma-\mathrm{FeSi}_{2}\left(\mathrm{CaF}_{2}\right.$ structure). We determine their lattice constants in order to better understand their existence as epitaxial phases on $\mathrm{Si}(111)$, and give a detailed account of their electronic band structures. We focus on the bonding between $\mathrm{Fe}$ and $\mathrm{Si}$ both in FeSi and $\gamma-\mathrm{FeSi}_{2}$ and compare to $\mathrm{NiSi}_{2}$ and $\mathrm{CoSi}_{2}$. Special attention is given to the covalent versus ionic nature of the Fe-Si bond, and the role of $\mathrm{Si} p-\mathrm{Fe} d$ interaction. 
The paper is organized as follows. In Section II we present the details of our ab initio calculations and the resulting equilibrium structural parameters of the two hypothetical bulk phases; the Fe-Si bond is studied in Sec. III; in Sec. IV the electronic band structures are discussed; and finally the conclusions are given in Sec. $\mathrm{Z}$.

\section{SELF-CONSISTENT CALCULATIONS}

FeSi is assumed in the $\mathrm{CsCl}$ structure (space group $\operatorname{Pm} \overline{3} m$ ), i.e., in a simple cubic lattice with $\mathrm{Fe}$ at the origin and $\mathrm{Si}$ at the body center. Both types of atoms are coordinated with 8 nearest neighbors $(n n)$ of the other species. $\gamma$-FeSi $i_{2}$ crystallizes in the $\mathrm{CaF}_{2}$ structure (space group $F m \overline{3} m$ ), i.e., in a face-centered cubic lattice with $\mathrm{Fe}$ at the origin, and two $\mathrm{Si}$ atoms at positions $\pm\left(\frac{1}{4} \frac{1}{4} \frac{1}{4}\right)$, respectively. Each Si atom is tetrahedrally coordinated with 4 metal atoms, and each metal atom has 8 Si $n n$.

We have calculated the electronic energy eigenvalues, total energies, and wave functions of the two crystals with the self-consistent full-potential linearized augmented planewave (FLAPW) method 16 , neglecting spin-polarization. The exchange-correlation energy is treated in the local density approximation (LDA), using the Hedin-Lundqvist prescription. Wave functions are expanded in spherical harmonics with $l \leq 8(l \leq 4$ for total energy minimization) inside the atomic spheres whose radii are 1.85 a.u. and 2.3 a.u. for $\mathrm{Si}$ and Fe, respectively. In the interstitial region a plane wave basis is used with wave vectors up to $3.6 \mathrm{Ry}$ (8.5 Ry) for wave functions (charge and potential). Integration in $\boldsymbol{k}$-space is approximated by summing over up to $48 \boldsymbol{k}$-points in the irreducible wedge of the corresponding Brillouin zone. The energy band structure and density of states (DOS) of the equilibrium structures are evaluated using higher cutoffs and more $\boldsymbol{k}$-points.

The lattice constants and bulk moduli obtained by total energy calculations are summarized in Tab. If, together with the lattice constants of the bulk phases. We have fitted the total energies calculated at six different lattice constants to both Murnaghan's equation of state 17 , and a third- or fourth-order polynomial. The predicted equilibrium lattice constant is independent of the fitting procedure, whereas the fluctuations of the fitted bulk modulus provide the theoretical error bars given in the Table. The theoretical values predict a virtually perfect lattice match of CsCl-type FeSi and Si. Recent X-ray diffraction datat on thin epitaxial FeSi films yield a lattice constant $a_{\mathrm{FeSi}}^{\exp }=2.77 \AA$, which is $1.8 \%$ larger than our theoretical value, and a larger lattice mismatch with $\mathrm{Si}$, i.e., $2 a_{\mathrm{FeSi}}^{\exp }=5.54 \AA\left(a_{\mathrm{Si}}^{\exp }=5.43\right.$ $\AA)$ which remains however rather small. The predicted lattice constant of $\gamma-\mathrm{FeSi}_{2}$ is only 2 $\%$ smaller than that of FeSi (Tab. 目), even though there are only half as many iron atoms per mole as in FeSi. Assuming the same magnitue of error on our predicted lattice constant as for the $\mathrm{CsCl}$ structure, we obtain a corrected value of $5.42 \AA$, which is almost perfectly lattice matched with Si. This explains why the fluorite phase can compete with the lattice mismatched bulk phase $\left(\beta-\mathrm{FeSi}_{2}\right)$ in the presence of the Si substrate (see our discussion in the Introduction). While the Fe-Si $n n$ distance differs by only $2 \%$ in the two crystals, the $\mathrm{Fe}-\mathrm{Fe} n n$ distance is $2.72 \AA$ in $\mathrm{FeSi}$, and $3.76 \AA$ in $\mathrm{FeSi}_{2}$. Note that this Fe-Fe spacing in FeSi is only $10 \%$ greater than the $n n$ distance, and $5 \%$ smaller than the next-nearest neighbor distance along the cube edge in $b c c$ iron.

Given the near lattice matching of both $\mathrm{FeSi}(\mathrm{CsCl})$ and $\mathrm{FeSi}_{2}\left(\mathrm{CaF}_{2}\right)$ with $\mathrm{Si}$, we shall describe the electronic properties of the unstrained hypothetical equilibrium phases, rather 
than the rhombohedrally distorted ones observed on $\mathrm{Si}(111)$. The strain induced changes on the electronic band structure are expected to be very small.

\section{THE Fe-Si BOND}

The nature of the chemical bond in transition metal silicides has been the subject of extensive theoretical and experimental studies 18 22. In general, the bonding in the silicides is understood in terms of metal $d$ - silicon $p$ hybridization. There has been a controversy about the bonding in $\mathrm{NiSi}_{2}$. Tersoff and Hamann 19 proposed that the bonding in $\mathrm{NiSi}_{2}$ and $\mathrm{CoSi}_{2}$ is similar to that in $\mathrm{Si}$, an idea first put forward by Chabal et al 18 Following their arguments, the tetrahedrally coordinated $\mathrm{Si}$ atoms build $s p^{3}$ hybrids, which in the silicides form directed covalent bonding states with Fe $d$ orbitals. In a later study, Lambrecht et al 21 came to a similar conclusion, but attributed more importance to the charge transfer from $\mathrm{Si}$ to $\mathrm{Ni}$ which contributes an electrostatic term to the cohesive energy. It is well known, however, that the charge transfer is not uniquely determined, since it depends on the choice of the "atomic spheres" where the charge is measured. With this in mind, we compare in Tab. II the valence charge contained in the atomic spheres used in our calculations. Besides the self-consistent density of the two crystals we have calculated the charge density of the isolated atoms as well as the lattice superpositions of the atomic densities. In $\mathrm{FeSi}_{2}$, the 8 Si $n n$ of Fe contribute more charge to the Fe spheres due to the smaller Fe-Si distance. On the other hand, the six Fe $n n$ of Fe in FeSi are much closer than the $12 \mathrm{Fe} n n$ of $\mathrm{Fe}$ in $\mathrm{FeSi}_{2}$, therefore superposition of atomic densities gives a Fe charge 0.08 electrons larger in FeSi than in $\mathrm{FeSi}_{2}$. As to the charge in the $\mathrm{Si}$ spheres it is obvious that twice the number of $\mathrm{Fe} n n$ of $\mathrm{Si}$ in FeSi yields a larger overlap than the corresponding one in $\mathrm{FeSi}_{2}$. The remaining free-atom charge is distributed in the interstitial region. When comparing the free-atom superpositions with the self-consistent densities, we observe an additional net charge transfer $\Delta Q_{\mathrm{Fe}}$ of 0.18 electrons into the $\mathrm{Fe}$ sphere in $\mathrm{FeSi}$, and one of 0.35 electrons into the $\mathrm{Fe}$ sphere in $\mathrm{FeSi}_{2}$. Note that now the Fe sphere in $\mathrm{FeSi}_{2}$ contains more charge than the one in FeSi. It would be misleading, however, to draw conclusions on the ionicity from these numbers alone, due to the ambiguity pointed out above. We will show that the excess charge in the $\mathrm{FeSi}_{2} \mathrm{Fe}$ sphere with respect to the FeSi one is due to a piling up of covalent charge in the Fe-Si bond region of $\mathrm{FeSi}_{2}$.

To this end we analyze the charge densities instead of the integrated charge in the spheres. In Fig. 2 we display contour plots of the difference between the self-consistent charge density and the superposition of atomic densities in the (110) plane of $\mathrm{FeSi}_{2}$ (a) and FeSi (b). Besides the obvious difference due to the presence of an additional Fe atom in $\mathrm{FeSi}$, we notice a weak piling up of charge midway between $\mathrm{Fe}$ and $\mathrm{Si}$ in the fluorite structure. This covalent charge accumulation is almost entirely contained in the Fe sphere, and is responsible for the larger charge transfer in $\mathrm{FeSi}_{2}$ discussed above. In $\mathrm{FeSi}$, on the other hand, the charge re-distribution is unimportant in the interstitial and bond region, and we interpret the charge transfer of 0.18 electrons from the Si to the Fe spheres as a real

ionic character of the bond. This conclusion will be reinforced by the crystal field arguments presented in the next section. 


\section{ELECTRONIC STRUCTURE}

The energy dispersion relations of $\gamma-\mathrm{FeSi}_{2}$ and $\mathrm{CsCl}$-type $\mathrm{FeSi}$ can be found in Refs. 4 and 5 , respectively, and are not reproduced here. In Fig. 3 we show the DOS of the two materials instead 23. In Tab. III we provide the angular-momentum projected densities at $E_{F}$ and integrated up to $E_{F}$, respectively. Our DOS for $\mathrm{FeSi}_{2}$ is in perfect agreement with the LMTO one by Christensent. The two main peaks in the valence spectrum originate from the metal $d / \mathrm{Si} p$ bonding states at $\sim-4 \mathrm{eV}\left(\Gamma_{25^{\prime}}^{v}\right.$ states at the zone center) and from the non-bonding metal $d$-band manifold centered at $\sim-1.5 \mathrm{eV}\left(\Gamma_{12}^{v}\right.$ states at the zone center), respectively. LDA energy eigenvalues at high symmetry points are listed in Tab. IV, together with the angular-momentum decomposition of the wave functions inside the atomic spheres. The $p d$ hybridization broadens the metal $d$ bands considerably as can be seen from the DOS and the orbital characters of the eigenfunctions. Given the strong similarity between the present $\mathrm{Si} s$ and $p$ partial DOS with those of $\mathrm{CoSi}_{2}{ }_{2} 19$, we conclude that the binding mechanism in $\mathrm{FeSi}_{2}$ is basically the same as in the former compound. It has been shown by Christensen that the rigid band assumption is nearly fulfilled for the three compounds $\mathrm{FeSi}_{2}, \mathrm{CoSi}_{2}$ and $\mathrm{NiSi}_{2}$ (see Fig. 1 in Ref. 1 ), and therefore the number of valence electrons pins the position of the Fermi level in an otherwise similar DOS. At the Fermi level there is a rather strong peak in the $d$-DOS of $\mathrm{FeSi}_{2}\left(28.5 \mathrm{Ry}^{-1}\right.$, cf. Tab. [III), with an appreciable admixture of Si $p$-states. The location in $\boldsymbol{k}$-space of these states is shown in Ref. Tt to be on the hexagonal Brillouin-zone face. We have previously estimated 6 that the Stoner factor is considerably larger than unity and explained bulk instability of the fluorite phase in terms of the Stoner model. Indeed, a spin-polarized calculation by Christensen predicts a ferromagnetic moment of $0.3 \mu_{B}$ per formula unit Experimental confirmation of ferromagnetism in $\gamma-\mathrm{FeSi}_{2}$ is still lacking, since the grown films are very thin $(\leq 15 \AA)$ and magnetic measurements are difficult to perform. Above $E_{F}$ we observe a Si $s p$ antibonding peak at $3 \mathrm{eV}$, which seems to be even more pronounced than in $\mathrm{CoSi}_{2} \mathrm{Ig}$, and which is a typical fingerprint of $s p^{3}$ hybridization. Together with the discussion of the charge transfer in Sec. [II] the picture of covalent bonding in fluorite $\mathrm{FeSi}_{2}$ is thus confirmed, similarly to the case of $\mathrm{CoSi}_{2}$ and $\mathrm{NiSi}_{2}$, and in agreement with the interpretation of Tersoff and Hamann ${ }^{19}$ for the latter two compounds. In $\mathrm{FeSi}_{2}$, however, the covalent contribution to the free energy is too small to stabilize the fluorite phase in bulk form. The kinetics and epitaxial constraints of iron silicide grown by MBE on $\operatorname{Si}(111)$, however, lead to the formation of fluorite $\mathrm{FeSi}_{2}$ as an intermediate step of the disordered $\mathrm{Fe}_{0.5} \square_{0.5} \mathrm{Si}$ to $\beta$ - $\mathrm{FeSi}_{2}$ transition均 at $\sim 500^{\circ} \mathrm{C}$.

Let us now turn to FeSi (right panel in Fig. 3, and Tab. У). Again the total DOS is dominated by the metal $d$ bands, giving rise to a strong $2 \mathrm{eV}$ broad peak centered at $\sim-2$ $\mathrm{eV}$. The Fermi level is located at the upper end of a region of low DOS, at the onset of a less pronounced, sharp peak of Fe $d$ states. The valence DOS agrees very well with the

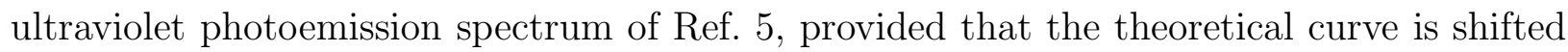
by $\sim 1 \mathrm{eV}$ towards $E_{F}$. This final-state effect is discussed in more detail in Ref. 5 . A very similar DOS is also predicted for CsCl-type RuSi by a LMTO Green's function calculation of Ivanovskii2 4 . There it is also shown that $\mathrm{RhSi}$ has a virtually identical DOS, except for the position of $E_{F}$ which now falls at higher energy in the sharp $d$ peak. The same behavior can therefore be anticipated when replacing $\mathrm{Fe}$ by $\mathrm{Co}$ in $\mathrm{FeSi}$, indicating that the hypothetical 
$\mathrm{CsCl}$ phase of CoSi is even less stable than FeSi, and likely to be magnetic. Below $E_{F}$ the $\mathrm{Si}$ $s$ and $p$ projected DOS is rather similar to that in $\mathrm{FeSi}_{2}$. This does not imply the formation of $s p^{3}$ hybrids, however, since $\mathrm{Si}$ is now 8-fold coordinated, instead of 4-fold as in the fluorite structure. We attribute the relatively large width of the Si $s$ band to bonding with Fe $s$ and $p$ states (see Tab. $\mathrm{\nabla}$ ).

An important difference between the band structures of the two materials lies in the order of the Fe $d$ bands at $\Gamma$ : in FeSi $\Gamma_{25^{\prime}}$ lies above $\Gamma_{12}$, whereas in $\mathrm{FeSi}_{2} \Gamma_{25^{\prime}}$ lies below $\Gamma_{12}$. An inverted crystal field splitting was previously noted in band calculations of chalcopyrites 25 $\left(\mathrm{CuInSe}_{2}\right)$ and in II-VI compounds2 2 . The results were explained in terms of symmetryenforced $p d$ couplings. A similar interpretation is pertinent here: In FeSi the Si $p$-bands $(x, y, z)$ transform like $\Gamma_{15}$, and thus do not mix with the $d$-band triplet $(x y, y z, z x)$ of $\mathrm{Fe}$, which transforms like $\Gamma_{25^{\prime}}$. Hence, the crystal field splitting corresponds to $\Gamma_{12}$ below $\Gamma_{25^{\prime}}$ like predicted by the point-ion model. This mixing is allowed, however, in the fluorite structure, where the $p$-orbitals of the two Si atoms in the unit cell originate both $\Gamma_{15}$ and $\Gamma_{25^{\prime}}$ states. The resulting $p d$ bonding lowers the $\Gamma_{25^{\prime}}$ level relative to the non-bonding $\Gamma_{12}$ level, thus reversing the sign with respect to that originating from the crystal field splitting alone. This effect is illustrated in Fig. 4 , where the energy levels of the Fe $d$ and Si $p$ states are shown for $\mathrm{FeSi}_{2}$ in the $\mathrm{CaF}_{2}$ structure, (a) without and (b) with taking the $p d$ interaction into account. The $p d$ repulsion found in certain II-VI zincblende semiconductors has a similar origin26: the cation $d$ bands of $\Gamma_{15}$ symmetry mix with the anion $p$ bands and are pushed below the $\Gamma_{12}$ $d$ states, opposed to crystal field considerations alone. Note, however, that the $p d$ repulsion in $\mathrm{ZnTe}, \mathrm{CdTe}$, and $\mathrm{HgTe}$, e.g., is much weaker than in the present case of $\mathrm{FeSi}_{2}$ and in the case of the chalcopyrites, since $(i)$ the cation $d$ - anion $p$ energy separation is larger for these atom pairs than for $\mathrm{Fe}$ and $\mathrm{Si},(i i)$ the cation-anion distance is considerably larger than the $\mathrm{Fe}-\mathrm{Si}$ one in $\mathrm{FeSi}_{2}$. Therefore, the $\Gamma_{15}-\Gamma_{12}$ splittings are much smaller in magnitude in these II-VI compounds 26 than the $\Gamma_{25^{\prime}}-\Gamma_{12}$ splitting in $\mathrm{FeSi}_{2}$, which we predict to be -2.55 $\mathrm{eV}$.

In $\mathrm{FeSi}$, on the other hand, $E\left(\Gamma_{25^{\prime}}\right)-E\left(\Gamma_{12}\right)=+3.24 \mathrm{eV}$, which is an unusually large crystal field splitting. We argue that it has two major contributions, an ionic one and one due to repulsion between Fe $d$ states of neighboring Fe atoms. Placing positive point charges +ze on the Si atom sites and negative charges - ze on the Fe sites will lower the electrostatic binding energy of the $\Gamma_{12}$ states relative to the $\Gamma_{25^{\prime}}$ ones. We estimate the ionic contribution $\Delta E_{i}$ to the splitting by assuming analytic Slater orbitals for the Fe $3 d$ wave functions, and by performing the lattice sum involved in the fourth-order non-spherical portion of the crystal potential as described by Callaway 27 for cubic crystals. We obtain $\Delta E_{i} \approx+1.14 z \mathrm{eV}$ for $\mathrm{Fe}^{-z} \mathrm{Si}^{+z}$. Clearly, this crude point-charge ionic model is not sufficient to quantitatively explain the level splitting, but the important points are that the sign agrees with the calculated one and that ionicity contributes to the splitting. A similar calculation for the fluorite structure (placing the charge $-2 z e$ on the Fe sites) yields a $d$ level splitting with equal sign as in FeSi, but $40 \%$ smaller. This is not surprising, since the nearest neighbor configuration of the Fe atoms is the same for both structures, and the contribution from the outer shells decreases rapidly 27. Hence, neglecting $p$ - $d$ bonding in the fluorite structure the $\Gamma_{12}$ states would be lower in energy than the $\Gamma_{25^{\prime}}$ ones (Fig. 四(a)), very much like in the $\mathrm{CsCl}$ structure. This shows once more that ionicity is not important in $\mathrm{FeSi}_{2}$.

The other effect which contributes to the large splitting in FeSi is the Fe-Fe interaction. 
We noted in Sec. [1] that the Fe-Fe $n n$ distance in $\mathrm{FeSi}$ is rather close to the equilibrium bond length in $b c c$ iron. According to Harrisones the $d d$ interatomic matrix elements decrease with the 5th power of the distance, and should therefore be about $60 \%$ as large as in $b c c$ iron. Recent tight-binding fits to the present band structures show indeed that the Fe-Fe $d d$ interaction cannot be neglected in $\mathrm{FeSi}$, whereas it is less important in ${ }^{29} \mathrm{FeSi}_{2}$. In $\mathrm{FeSi}$ the Fe atoms occupy simple cubic lattice points, and hence are octahedrally coordinated to their nearest Fe neighbors. The $\Gamma_{12}$ orbitals form $\sigma$-bonds, whereas the $\Gamma_{25^{\prime}}$ orbitals are $\pi$-bonded. Using Harrison's universal tight-binding parameter $2^{22}$ a splitting of $\sim 2.2 \mathrm{eV}$ is obtained. Again, this is not sufficient by itself to explain the splitting obtained in the self-consistent calculation. Considering in addition the ionic contribution discussed above, however, (calculated with a better approximation than the point-charge model) one should recover the $\Gamma_{25^{\prime}}-\Gamma_{12}$ splitting of the FLAPW calculation.

Inspection of Tab. $\mathrm{Q}$ and of Fig. 3 (right panel) shows that $\mathrm{Si} p / \mathrm{Fe} d$ hybridization is present also in $\mathrm{FeSi}$, although to a smaller extent than in $\mathrm{FeSi}_{2}$, at $\boldsymbol{k}$-points other than $\Gamma$. At the $R$-point, e.g., the $\boldsymbol{k}$-dependent phase factors associated with the atomic orbitals in a LCAO scheme cause the Si $(x, y, z)$ - like and the Fe $(x y, y z, z x)$ - like wave functions to belong to the same irreducible representation $R_{15}$, as they do under the $T_{d}$ point group. Also along the $\Lambda$-line $p d$ hybridiztion is allowed by symmetry, and leads to anticrossing (see Fig. 4 in Ref. 5) of the twofold degenerate $\Lambda_{3}$ bands originating from both $\Gamma_{12}$ and $\Gamma_{25^{\prime}}$ (retaining only $d d$ matrix elements, these bands would cross along $\Lambda$ ). Due to $p d$-bonding the $R_{15}$ state is pushed below $\Gamma_{12}$, and contributes to the peak in the DOS at $\sim-6 \mathrm{eV}$ (see Fig. (3). The upper $\Lambda_{3}$ band ends in a " $d d$-antibonding" $R_{12}^{c}$ state close to the Fermi level, with $99 \% d$-character in the Fe atomic spheres. Due to the strong $d d$ interaction we refrain from using the term "non-bonding $d$ " for these states.

\section{CONCLUSIONS}

We have presented the first theoretical study of the electronic and structural properties of the bulk unstable cubic iron silicide $\mathrm{FeSi}(\mathrm{CsCl})$, and have compared them with those of $\gamma-\mathrm{FeSi}_{2}\left(\mathrm{CaF}_{2}\right)$. Both structures exist epitaxially on $\mathrm{Si}(111)$ due to a lattice mismatch with $\mathrm{Si}$ wich is smaller than that of the bulk stable phases $\epsilon$ - $\mathrm{FeSi}$ and $\beta$-FeSi $\mathrm{F}_{2}$, respectively. In addition, kinetic barriers are believed to exist which account for the observed metastability of $\mathrm{FeSi}(\mathrm{CsCl})$ films at thicknesses exceeding its epitaxial stability range (Fig. 1).

We have established the close resemblance of the bonding configuration of $\mathrm{FeSi}_{2}$ with $\mathrm{CoSi}_{2}$ and $\mathrm{NiSi}_{2}$, i.e., Si $s p^{3}$ hybridization, strong metal $d / \mathrm{Si} p$ bonding, the presence of directed covalent Fe-Si bonds, and negligible ionicity. The giant density of $d$ states at the Fermi level leads to a Jahn-Teller instability of the bulk compound, explaining its absence in the bulk phase diagram.

CsCl-type $\mathrm{FeSi}$, on the other hand, is characterized by a small charge transfer from $\mathrm{Si}$ to Fe, strong Fe-Fe $d d$ bonding, and to some extent also by Si $p /$ metal $d$ interaction. We have shown that both the charge distribution and the electronic band structure of the two materials are consistent with the bonding mechanisms presented in this work. An empirical tight-binding study of the Fe silicides is under wayeg, and will address the lattice dynamics and its implications on the $\gamma \rightarrow \beta$ phase transition in epitaxial $\mathrm{FeSi}_{2} / \mathrm{Si}(111)$. 
Comparison with other known monosilicides in the $\mathrm{CsCl}$ structure has allowed us to speculate on the possible existence of epitaxial $\mathrm{CoSi}(\mathrm{CsCl})$ on $\mathrm{Si}(111)$. Subsequent experiments have confirmed the epitaxial stability of this phase. A rigid-band model implies a rather high $d$-DOS at the Fermi level, which may cause the compound to order magnetically.

The disordered $\mathrm{CsCl}$ phase $\mathrm{Fe}_{1-x} \square_{x} \mathrm{Si}$ is observed over a wide range of Fe vacancy concentrations $0<x \leq 0.5$. From the present study of the ordered end compounds FeSi and $\mathrm{FeSi}_{2}$ we presume that the ionic, or Madelung contribution to the cohesive energy (present in the $\mathrm{CsCl}$, but not in the $\mathrm{CaF}_{2}$ phase) stabilizes the epitaxial disordered $\mathrm{Fe}_{0.5} \square_{0.5} \mathrm{Si} \mathrm{CsCl}$ structure relative to the ordered $\mathrm{CaF}_{2}$ structure. In the latter the Fe atoms are located on alternate $\{111\}$ planes, and the Si atoms are tetrahedrally coordinated with the Fe atoms. The role of the $d$ electrons, however, cannot be obtained from the properties of the end compounds alone. A rigid-band model would predict the Fermi level to fall in a region of high $d$-DOS for increasing $x$, which is hardly reconciled with the experimentally observed epitaxial stability and absence of magnetic order in the defect $\mathrm{CsCl}$ phase. Further theoretical studies using, e.g., the coherent phase approximation, are needed for a complete understanding of the electronic properties of $\mathrm{Fe}_{1-x} \square_{x} \mathrm{Si}$ as a function of $x$. We hope that the present study, which adds to the general understanding of transition metal silicides, will stimulate further theoretical and experimental work on the fascinating iron silicide system.

\section{ACKNOWLEDGMENTS}

The authors are indebted to M. Posternak for the permission to use his FLAPW code, to F. Hulliger for drawing their attention to the existence of CsCl-type mono-silicides other than $\mathrm{FeSi}$, and to A. Zunger and S. Froyen for a critical reading of the manuscript and helpful comments. Financial support by the Board of the Swiss Federal Institutes of Technology, and in part by the "Stiftung Entwicklungsfonds Seltene Metalle" is gratefully acknowledged. The calculations have been performed on the Cray Y-MP at ETH Zürich. 


\section{REFERENCES}

* Also at Institut de Physique Appliquée, Ecole Polytechnique Fédérale, Lausanne, Switzerland.

Present address: National Renewable Energy Laboratory, 1617 Cole Boulevard, Golden CO 80401-3393.

$\dagger$ Also at Department of Theoretical Physics and INFM, University of Trieste, I-34014 Trieste, Italy.

${ }^{1}$ H. von Känel, Mat. Sci. Rep. 8, 193 (1992).

${ }^{2}$ Atlas of Crystal Structure Types for Intermetallic Phases, eds. J. L. C. Daams, P. Villars, and J. H. N. van Vucht, (ASM International, Materials Park OH, 1991).

${ }^{3}$ Binary Alloy Phase Diagrams, ed. T. B. Massalski, vol. 2, p. 1108, (ASM International, Materials Park OH, 1986).

${ }^{4}$ N. E. Christensen, Phys. Rev. B 42, 7148 (1990).

${ }^{5}$ H. von Känel, K. A. Mäder, E. Müller, N. Onda, and H. Sirringhaus, Phys. Rev. B 45, 13807 (1992).

${ }^{6}$ N. Onda, J. Henz, E. Müller, K. A. Mäder, and H. von Känel, Appl. Surf. Sci. 56-58, 421 (1992); N. Onda, J. Henz, E. Müller, H. von Känel, C. Schwarz, and R. E. Pixley, Helv. Phys. Acta 64, 197 (1991); H. von Känel, R. Stalder, H. Sirringhaus, N. Onda, and J. Henz, Appl. Surf. Sci. 53, 196 (1991).

${ }^{7}$ N. Onda, H. Sirringhaus, S. Goncalves-Conto, C. Schwarz, E. Müller-Gubler, and H. von Känel, Mat. Res. Soc. Symp. Proc., in press, (1993).

${ }^{8}$ D. M. Wood and A. Zunger, Phys. Rev. B 40, 4062, (1989); A. Zunger and D. M. Wood, J. Cryst. Growth 98, 1 (1989).

${ }^{9}$ R. Bruinsma and A. Zangwill, J. Phys. (Paris) 47, 2055 (1986).

${ }^{10}$ H. Sirringhaus, N. Onda, E. Müller-Gubler, P. Müller, R. Stalder, and H. von Känel, Phys. Rev. B 47, 10567 (1993).

${ }^{11}$ L. N. Finnie, J. Less-Common Metals 4, 24 (1962).

${ }^{12}$ P. Villars, J. Less-Common Metals 92, 215 (1983).

${ }^{13}$ C. Schwarz, S. Goncalves-Conto, H. Sirringhaus, and H. von Känel, unpublished.

${ }^{14}$ R. Eppenga, J. Appl. Phys. 68, 3027 (1990).

${ }^{15}$ J. Kudrnovský, N. E. Christensen, and O. K. Andersen, Phys. Rev. B 43, 5924 (1991).

${ }^{16}$ H. Jansen and A. J. Freeman, Phys. Rev. B 30, 561 (1984).

${ }^{17}$ F. D. Murnaghan, Proc. Nat. Acad. Sci. USA 30, 244 (1944).

${ }^{18}$ Y. J. Chabal, D. R. Hamann, J. E. Rowe, and M. Schlüter, Phys. Rev. B 25, 7598 (1982).

${ }^{19}$ J. Tersoff and D. R. Hamann, Phys. Rev. B 28, 1168 (1983).

${ }^{20}$ O. Bisi and C. Calandra, J. Phys. C 14, 5479 (1981).

${ }^{21}$ W. R. L. Lambrecht, N. E. Christensen, and P. Blöchl, Phys. Rev. B 36, 2493 (1987).

${ }^{22}$ J. H. Weaver, A. Franciosi, and V. L. Moruzzi, Phys. Rev. B 29, 3293 (1984).

${ }^{23}$ Note that the DOS presented here is based on the LDA. Self-energy corrections are known to shift energy eigenvalues below $E_{F}$ to lower energies in proportion to the degree of their spatial localization. Thus, $d$ states will move more than $s p$ states. These corrections should not affect our conclusions, however.

${ }^{24}$ A. L. Ivanovskii, Izv. Akad. Nauk SSSR, Neorg. Mater. 26, 1226 (1990); English translation: Inorganic Materials (Plenum, New York, 1990).

${ }^{25}$ J. E. Jaffe and A. Zunger, Phys. Rev. B 28, 5822 (1983). 
${ }^{26}$ S.-H. Wei and A. Zunger, Phys. Rev. B 37, 8958 (1988).

27 J. Callaway, Quantum Theory of the Solid State (Academic, Boston, 1991), 2nd ed., p. 221.

${ }^{28}$ W. A. Harrison, Electronic Structure and the Properties of Solids (Freeman, San Francisco, $1980)$.

${ }^{29}$ G. Malegori and L. Miglio, private communication. 


\section{FIGURES}

FIG. 1. Schematic diagram of eptiaxial energy versus film thickness for FeSi on Si(111). The $\mathrm{CsCl}$ structure is more closely matched to the Si substrate than the bulk stable $\epsilon$-phase, and hence is epitaxially stable at small film thickness. Above its critical thickness $H_{c} \epsilon$-FeSi is the most stable phase, and the occurrence of the $\mathrm{CsCl}$ phase in this range can be explained by the existence of a kinetic barrier. Upon annealing at $\sim 300^{\circ} \mathrm{C}$ the $\mathrm{CsCl}$ phase transforms irreversibly into the bulk $\epsilon$-phase.

FIG. 2. Contour plot of the difference between the self-consistent charge density and the superposition of atomic densities in the (110) plane of $\mathrm{CaF}_{2}$-type $\mathrm{FeSi}_{2}$ (a) and CsCl-type FeSi (b). Areas of negative values are shaded, contours are labeled in units of $10^{-2}$ electrons $/(\mathrm{bohr})^{3}$, and the level spacing is 0.02 in these units. $\Delta Q_{\alpha}$ is the difference between the self-consistent valence charge and the superposition of the atomic valence charges in the sphere centered on atom $\alpha$. The Fe-Si bonds are indicated with solid lines.

FIG. 3. DOS of $\mathrm{CaF}_{2}$-type $\mathrm{FeSi}_{2}$ (left panel) and CsCl-type FeSi (right panel). (a) and (c) show the total (solid line) and partial Fe $d$ (dashed-dotted line) DOS in $\mathrm{eV}^{-1}$ per unit cell, (b) and (d) show the partial Si $p$ (solid line) and Si $s$ (dashed line) DOS. The partial DOS are projected on one atomic sphere in both structures. The energy is measured relative to the Fermi level (indicated by a solid vertical line).

FIG. 4. Energy levels of Fe $d$ and $\mathrm{Si} p$ bands at $\Gamma$ in the $\mathrm{CaF}_{2}$ structure of $\mathrm{FeSi}_{2}$, (a) neglecting, (b) including Fe $d / \mathrm{Si} p$ interaction. Note that $p d$ repulsion pushes the $\Gamma_{25^{\prime}}$ states below the $\Gamma_{12}$ states, whereas crystal field splitting alone (a) predicts the $\Gamma_{12}$ states to be lowest. 


\section{TABLES}

TABLE I. Structural definition of the stable bulk phasest of $\mathrm{FeSi}$ and $\mathrm{FeSi}_{2}$, as well as of the bulk unstable phases which exist epitaxially on $\mathrm{Si}(111)$. For the latter the theoretical predictions of the present work are compared to experimental values, where available, and to other theoretical results. The experimental lattice constant of $\mathrm{Si}$ is $5.43 \AA$.

\begin{tabular}{|c|c|c|c|c|}
\hline $\begin{array}{l}\text { Compound } \\
\text { (Pearson Symbol) }\end{array}$ & Space group & $\begin{array}{l}\text { Lattice } \\
\text { (exp.) }\end{array}$ & $\begin{array}{l}\text { heters }(\AA) \\
\quad(\text { theo. })\end{array}$ & $\begin{array}{c}\text { Bulk modulus (Mbar) } \\
\text { (theo.) }\end{array}$ \\
\hline $\begin{array}{l}\text { Bulk stable: } \\
\epsilon-\mathrm{FeSi} \\
(c P 8)\end{array}$ & $P 2_{1} 3$ & $a=4.46$ & & \\
\hline $\begin{array}{l}\beta-\mathrm{FeSi}_{2} \\
(o C 48)\end{array}$ & Cmca & $\begin{array}{l}a=9.86 \\
b=7.79 \\
c=7.83\end{array}$ & & \\
\hline $\begin{array}{l}\text { Bulk unstable: } \\
\text { FeSi }(\mathrm{CsCl}) \\
(c P 2)\end{array}$ & $P m \overline{3} m$ & $2 a=5.54^{\mathrm{a}}$ & $2 a=5.44$ & $2.70 \pm 0.07$ \\
\hline $\begin{array}{l}\mathrm{Fe}_{1-x} \square_{x} \mathrm{Si} \\
(\mathrm{CsCl}, x \leq 0.5) \\
\gamma-\mathrm{FeSi}_{2}\left(\mathrm{CaF}_{2}\right) \\
(c F 12)\end{array}$ & $\begin{array}{l}P m \overline{3} m \\
F m \overline{3} m\end{array}$ & $\begin{aligned} 2 a & =5.40^{\mathrm{a}} \\
(x & =0.5)\end{aligned}$ & $\begin{array}{l}a=5.32 \\
a=5.39^{b}\end{array}$ & $\begin{array}{c}1.90 \pm 0.05 \\
2.06^{\mathrm{b}}\end{array}$ \\
\hline
\end{tabular}

${ }^{a}$ Reference 7 .

${ }^{\mathrm{b}}$ Reference 1 .

TABLE II. Valence charge contained in the atomic spheres of radius $R$ and in the interstitial volume of CsCl-type $\mathrm{FeSi}$ and $\mathrm{CaF}_{2}$-type $\mathrm{FeSi}_{2}$. The total valence charge of $\mathrm{Fe}$ is 8 , and that of $\mathrm{Si}$ is 4 electrons.

\begin{tabular}{lllccc}
\hline \hline & Charge & Fe & Si & Interstitial & Total \\
& & $R=2.3$ a.u. & $R=1.85$ a.u. & & \\
\hline $\mathrm{Fe}, \mathrm{Si}$ & isolated atoms & 6.24 & 1.25 & & \\
$\mathrm{FeSi}$ & free-atom superposition & 7.30 & 1.66 & 3.04 & 12.0 \\
& self-consistent & 7.48 & 1.48 & 3.04 & 12.0 \\
$\mathrm{FeSi}_{2}$ & free-atom superposition & 7.22 & 1.55 & 5.68 & 16.0 \\
& self-consistent & 7.57 & 1.42 & 5.59 & 16.0 \\
\hline \hline
\end{tabular}


TABLE III. Partial number of states (NOS) below the Fermi energy $E_{F}$, and density of states at $E_{F}\left(\mathrm{DOS}\right.$ in $\mathrm{Ry}^{-1}$ ) normalized to one atomic sphere (cf. Tab. II) in CsCl-type FeSi and $\mathrm{CaF}_{2}$-type $\mathrm{FeSi}_{2}$.

\begin{tabular}{|c|c|c|c|c|c|c|c|}
\hline & & Fe $s$ & Fe $p$ & Fe $d$ & Si $s$ & Si $p$ & Si $d$ \\
\hline \multirow[t]{2}{*}{$\overline{\mathrm{FeSi}}$} & DOS & 0.001 & 0.018 & 1.678 & 0.051 & 0.020 & 0.004 \\
\hline & NOS & 0.385 & 0.530 & 6.528 & 0.599 & 0.720 & 0.143 \\
\hline \multirow[t]{2}{*}{$\mathrm{FeSi}_{2}$} & DOS & 0.317 & 3.740 & 28.478 & 0.073 & 2.115 & 0.449 \\
\hline & NOS & 0.466 & 0.540 & 6.544 & 0.607 & 0.706 & 0.099 \\
\hline
\end{tabular}

TABLE IV. FLAPW energy eigenvalues at high symmetry points of the face centered cubic Brillouin zone for $\mathrm{FeSi}_{2}$ in the $\mathrm{CaF}_{2}$ structure. Energies are measured relative to the Fermi level. The probability of the orbital character of the wavefuntions inside the Fe and Si atomic spheres is given in percent. In the last column we list the probability in the interstitial region.

\begin{tabular}{|c|c|c|c|c|c|c|c|c|}
\hline \multirow[t]{2}{*}{ State } & \multirow{2}{*}{$\begin{array}{c}\text { Energy } \\
(\mathrm{eV})\end{array}$} & \multicolumn{3}{|c|}{$\mathrm{Fe}$} & \multicolumn{3}{|c|}{$\mathrm{Si}$} & \multirow[t]{2}{*}{ int. } \\
\hline & & $\mathrm{s}$ & $\mathrm{p}$ & d & $\mathrm{s}$ & $\mathrm{p}$ & $\mathrm{d}$ & \\
\hline$\overline{\Gamma_{1}^{v}}$ & -13.07 & 14 & & & 30 & & & 56 \\
\hline$\Gamma_{25^{\prime}}^{v}$ & -3.95 & & & 50 & & 17 & & 32 \\
\hline$\Gamma_{12}^{v}$ & -1.40 & & & 80 & & & 3 & 17 \\
\hline$\Gamma_{2^{\prime}}^{c}$ & 0.87 & & & & 67 & & & 29 \\
\hline$\Gamma_{15}^{c}$ & 3.85 & & 28 & & & 2 & 13 & 56 \\
\hline$X_{1^{\prime}}^{v}$ & -8.93 & & 10 & & 36 & & 1 & 52 \\
\hline$X_{1}^{v}$ & -7.09 & 15 & & 8 & & 19 & & 58 \\
\hline$X_{3}^{v}$ & -6.43 & & & 28 & 33 & & 2 & 37 \\
\hline$X_{5}^{v}$ & -3.49 & & 13 & & & 27 & & 59 \\
\hline$X_{1}^{v}$ & -1.44 & 14 & & 52 & & 1 & 5 & 28 \\
\hline$X_{5}^{v}$ & -1.43 & & & 86 & & 4 & 1 & 9 \\
\hline$X_{2}^{v}$ & -0.67 & & & 91 & & & & 8 \\
\hline$X_{1}^{c}$ & 2.60 & & & 74 & 16 & & & 10 \\
\hline$L_{1}^{v}$ & -9.73 & 13 & & 5 & 26 & 5 & & 51 \\
\hline$L_{2^{\prime}}^{v}$ & -9.16 & & 10 & & 26 & 6 & & 58 \\
\hline$L_{3}^{v}$ & -3.41 & & & 53 & & 13 & 1 & 32 \\
\hline$L_{1}^{v}$ & -1.70 & 9 & & 63 & 2 & 5 & 2 & 17 \\
\hline$L_{3}^{v}$ & -1.04 & & & 87 & & & 1 & 11 \\
\hline$L_{3^{\prime}}^{v}$ & -0.24 & & 16 & & & 26 & 4 & 53 \\
\hline$L_{2^{\prime}}^{c}$ & 2.74 & & & & 23 & 11 & 3 & 59 \\
\hline
\end{tabular}


TABLE V. FLAPW energy eigenvalues at high symmetry points of the simple cubic Brillouin zone for $\mathrm{FeSi}$ in the $\mathrm{CsCl}$ structure. Energies are measured relative to the Fermi level. The probability of the orbital character of the wavefuntions inside the Fe and $\mathrm{Si}$ atomic spheres is given in percent. In the last column we list the probability in the interstitial region.

\begin{tabular}{|c|c|c|c|c|c|c|c|c|}
\hline \multirow[t]{2}{*}{ State } & \multirow{2}{*}{$\begin{array}{c}\text { Energy } \\
(\mathrm{eV})\end{array}$} & \multicolumn{3}{|c|}{$\mathrm{Fe}$} & \multicolumn{3}{|c|}{$\mathrm{Si}$} & \multirow[t]{2}{*}{ int. } \\
\hline & & $\mathrm{s}$ & $\mathrm{p}$ & d & $\mathrm{s}$ & $\mathrm{p}$ & $\mathrm{d}$ & \\
\hline$\overline{\Gamma_{1}^{v}}$ & -14.60 & 24 & & & 27 & & & 48 \\
\hline$\Gamma_{12}^{v}$ & -4.12 & & & 76 & & & 3 & 20 \\
\hline$\Gamma_{25^{\prime}}^{v}$ & -0.88 & & & 95 & & & 1 & 3 \\
\hline$\Gamma_{15}^{c}$ & 3.38 & & 17 & & & 37 & & 40 \\
\hline$R_{15}^{v}$ & -6.30 & & & 50 & & 16 & & 34 \\
\hline$R_{1^{\prime}}^{v}$ & -1.27 & & & & 64 & & & 30 \\
\hline$R_{12}^{c}$ & 0.07 & & & 99 & & & & 1 \\
\hline$R_{15}^{c}$ & 1.67 & & 39 & & & & 12 & 48 \\
\hline$X_{2^{\prime}}^{v}$ & -10.47 & & 17 & & 34 & & & 48 \\
\hline$X_{1}^{v}$ & -9.04 & 26 & & 10 & & 17 & & 46 \\
\hline$X_{3}^{v}$ & -3.15 & & & 87 & & & & 12 \\
\hline$X_{5}^{v}$ & -2.53 & & & 81 & & 6 & & 11 \\
\hline$X_{4}^{v}$ & -0.69 & & & 97 & & & & 2 \\
\hline$X_{3}^{c}$ & 0.34 & 4 & & 89 & & 2 & & 4 \\
\hline
\end{tabular}

